\title{
Sondage sérologique de la fasciolose bovine en Guinée
}

\author{
A.M. Barry ${ }^{1}$ S. Keita ${ }^{1}$
}

\section{Mots-clés}

Bovin - Fasciola gigantica Fasciolose - Test Elisa - Guinée.

\section{Résumé}

Un sondage sérologique sur la fasciolose a été réalisé en Guinée sur des sérums issus de la sérothèque du Laboratoire de diagnostic vétérinaire de la Direction nationale des services vétérinaires. Au total, 480 sérums provenant de bovins N’Dama ont été sélectionnés par randomisation et analysés par la technique de dosage immuno-enzymatique (Elisa). Les résultats obtenus ont mis en évidence la présence d'anticorps de douves chez 44,1 p. 100 des bovins, avec un taux de 35,5 p. 100 dans la région Ouest de la Guinée et de 55,5 p. 100 dans la région Est. Les bovins de tous les âges ont été séropositifs avec des taux variant de 27,8 à 44,5 p. 100 .

\section{INTRODUCTION}

La fasciolose bovine est une helminthose du foie provoquée par des trématodes du genre Fasciola et dont l'espèce $F$. gigantica a été décrite en Afrique occidentale. Pour sa mise en évidence, la coprologie demeure la seule méthode de diagnostic ante mortem. Cette méthode est insuffisante car elle ne met les œufs en évidence qu'à la dixième semaine (5). En revanche, l'analyse immunologique par la technique de dosage immuno-enzymatique (Elisa) permet de trouver les premiers anticorps de la fasciolose dès la troisième semaine après l'infestation (3). L'Elisa a été largement adopté dans les études sérologiques de la fasciolose $(5,8,9)$; cet outil est approprié pour les études épidémiologiques de cette

1. Direction nationale des services vétérinaires, BP 559, Conakry, Guinée.

Tél. : + 22460513006 ; e-mail : abarrymadiou@yahoo.fr

Tél. : + 22460436107 ; e-mail : K.sory@yahoo.fr maladie (2). Au Sénégal, un test Elisa utilisant des antigènes de $F$. hepatica a permis de mettre en évidence des anticorps de douves dans des sérums de bovins. Il a une sensibilité de 98-99 p. 100 et une spécificité de 98-100 p. 100 (6). L'Elisa est sensible et spécifique en utilisant aussi comme antigène les produits d'excrétion et de sécrétion de $F$. gigantica (3). Les valeurs de sensibilité et de spécificité du test Elisa pour ces produits de $F$. gigantica chez les bovins sont respectivement de 93,3 et 96,7 p. 100 (1). Il existe une communauté antigénique entre $F$. hepatica et $F$. gigantica (7).

La fasciolose est présente en Guinée. Dans les abattoirs, 8 p. 100 des animaux abattus présentent des douves du foie (10). Peu d'informations existent sur la répartition de la maladie. L'objectif de cette étude a été de faire un sondage sur la sérothèque du Laboratoire de diagnostic vétérinaire de la Direction nationale des services vétérinaires pour connaître la prévalence de la fasciolose bovine en Guinée et essayer, dans une seconde étape, de faire une enquête épidémiologique plus approfondie dans la région à forte prévalence de la maladie. 


\section{MATERIEL ET METHODES}

La Guinée est divisée en régions, préfectures, sous-préfectures et villages. Dans les villages, les bovins de race N'Dama sont regroupés en troupeaux villages. La taille moyenne du troupeau village est de 50 têtes. La sérothèque du laboratoire a été constituée en utilisant le troupeau comme unité d'échantillonnage. Dans chaque unité, des échantillons sanguins ont été prélevés, au niveau de la veine jugulaire en utilisant des tubes secs, sur un tiers des animaux (15 animaux). Après sédimentation des hématies, les sérums obtenus ont été aliquotés dans des cryotubes numérotés, puis congelés et conservés à $-20^{\circ} \mathrm{C}$.

La sérothèque a été constituée en 1991. En fonction de la densité du cheptel de chaque région, la sérothèque contient $16,4,40,6$, 34,5 et 8,4 p. 100 de sérums provenant respectivement de la Basse Guinée, de la Moyenne Guinée, de la Haute Guinée et de la Guinée forestière. L'ensemble de la sérothèque comprend 5500 sérums. De cet ensemble 90 (1,6 p. 100), 183 (3,3 p. 100), 157 (2,8 p. 100) et $50(0,9$ p. 100) sérums provenant respectivement des régions citées, soit 480 sérums ou 8,7 p. 100, ont été sélectionnés par randomisation. Ainsi, 480 nombres aléatoires correspondant aux numéros des cryotubes ont été sélectionnés. Ces échantillons ont été décongelés, puis analysés par Elisa. Ils provenaient de 62 troupeaux villageois.

La saisie et l'analyse des données ont été effectuées par le logiciel Epi-Info version 6.0. Un test du chi ${ }^{2}$ a été utilisé, d'une part, pour comparer les proportions d'infestations des régions Est et Ouest de la Guinée et, d'autre part, pour apprécier l'effet du sexe sur l'infestation.

Le kit de détection de la fasciolose hépatique utilisé a été de type Elisa (fabricant Beldico, Belgique ; distributeur Sanofi Diagnostic Pasteur). Une culture de l'antigène parasitaire est adsorbée dans les puits de la microplaque. Les anticorps bovins spécifiques du parasite sont mis en évidence par un anticorps monoclonal antiimmunoglobuline $\mathrm{G} 1$ ( $\mathrm{IgG} 1)$ bovin couplé à la peroxydase. La formation des immunocomplexes est révélée par l'apparition d'une coloration bleue. L'intensité de la coloration résultant de l'activité enzymatique est proportionnelle à la teneur en anticorps spécifiques de l'échantillon sérique. Les résultats sont évalués à l'aide d'un lecteur Elisa utilisant un filtre de $450 \mathrm{~nm}$.

Selon le fabricant, un échantillon est positif si son coefficient est supérieur à 0,2 . Ce coefficient est calculé en utilisant la formule :

Coef. $=\frac{\mathrm{DO}_{\text {échantillon }}-\mathrm{DO}_{\text {sérum négatif }}}{\mathrm{DO} \mathrm{Sérum} \mathrm{positif}_{\text {sé }}-\mathrm{DO}_{\text {sérum négatif }}}$

où $\mathrm{DO}$ est la densité optique.

\section{RESULTATS}

Au total, 480 sérums provenant de 25 préfectures ont été analysés. Le pourcentage global de réponses positives a été de 44,1 p. 100 (tableau I).

A l'Est de la Guinée, 55,5 p. 100 des animaux ont été séropositifs contre 35,5 p. 100 à l'Ouest (différence significative ; $\mathrm{chi}^{2}=38,7$; $\left.\mathrm{ddl}=1 ; \mathrm{p}<10^{-3}\right)$ (tableau II).

Les répartitions par sexe et par âge sont présentées respectivement dans les tableaux III et IV.

L'infestation des troupeaux villages par les douves est montrée dans le tableau $\mathrm{V}$.
Tableau I

Séroprévalence de l'infestation à Fasciola sp. chez les bovins N'Dama dans les différentes préfectures de la Guinée

\begin{tabular}{|c|c|c|c|c|}
\hline \multirow[b]{2}{*}{ Région } & \multicolumn{4}{|c|}{ Elisa } \\
\hline & Préfecture & Total & Positifs & $\%$ \\
\hline \multirow[t]{7}{*}{ Basse Guinée } & Boffa & 8 & 1 & \\
\hline & Boké & 25 & 7 & \\
\hline & Coyah & 8 & 3 & \\
\hline & Forécariah & 9 & 0 & \\
\hline & Fria & 2 & 0 & \\
\hline & Kindia & 29 & 13 & \\
\hline & Télimélé & 9 & 2 & \\
\hline Sous-total & & 90 & 26 & 28,8 \\
\hline \multirow[t]{8}{*}{ Moyenne Guinée } & Gaoual & 47 & 17 & \\
\hline & Koubia & 10 & 6 & \\
\hline & Koundara & 33 & 9 & \\
\hline & Labé & 5 & 0 & \\
\hline & Mamou & 23 & 13 & \\
\hline & Pita & 10 & 0 & \\
\hline & Tougué & 10 & 3 & \\
\hline & Mali & 45 & 23 & \\
\hline Sous-total & & 183 & 71 & 38,7 \\
\hline \multirow[t]{7}{*}{ Haute Guinée } & Dabola & 10 & 7 & \\
\hline & Dinguiraye & 29 & 11 & \\
\hline & Faranah & 36 & 17 & \\
\hline & Kankan & 26 & 12 & \\
\hline & Kérouane & 19 & 14 & \\
\hline & Mandiana & 6 & 3 & \\
\hline & Siguiri & 31 & 11 & \\
\hline Sous-total & & 157 & 75 & 47,7 \\
\hline \multirow[t]{3}{*}{ Guinée forestière } & Beyla & 10 & 8 & \\
\hline & Guéckedou & 10 & 3 & \\
\hline & Kissidougou & 30 & 29 & \\
\hline Sous-total & & 50 & 40 & 80,0 \\
\hline Total & & 480 & 212 & 44,1 \\
\hline
\end{tabular}

Tableau II

Séroprévalence de l'infestation à Fasciola sp. chez les bovins N’Dama dans les différentes régions de la Guinée

\begin{tabular}{|c|c|c|}
\hline Région & Nb. positifs & $\%$ positifs \\
\hline Ouest $(B G, M G)^{a}$ & 97 & 35,5 \\
\hline Est $(\mathrm{HG}, \mathrm{GF})^{b}$ & 115 & 55,5 \\
\hline Total & 212 & 44,1 \\
\hline
\end{tabular}

BG : Basse Guinée ; MG : Moyenne Guinée HG : Haute Guinée ; GF : Guinée forestière a, ${ }^{b}$ chi $^{2}=38,78 ; \mathrm{ddl}=1 ; \mathrm{p}<10^{-3}$ 


\section{Tableau III}

Séroprévalence de l'infestation à Fasciola sp. chez les bovins N'Dama par sexe

\begin{tabular}{lcc} 
Sexe & Nb. positifs & \% positifs \\
\hline Mâle $^{\text {a }}$ & 58 & 45,3 \\
Femelle $^{b}$ & 153 & 43,5 \\
Total & 211 & 44,0
\end{tabular}

a,b chi $^{2}=0,27 ; \mathrm{ddl}=1 ; \mathrm{p}=0,6$

\section{Tableau IV}

Séroprévalence de l'infestation à Fasciola sp. chez les bovins N'Dama par âge

\begin{tabular}{lccc} 
Age & Nb. sérums & Nb. positifs & \% positifs \\
\hline$\leq 1$ an & 61 & 17 & 27,8 \\
$1-2$ ans & 21 & 11 & 52,3 \\
$2-3$ ans & 54 & 27 & 50,0 \\
$3-4$ ans & 43 & 19 & 44,1 \\
$4-5$ ans & 61 & 31 & 50,8 \\
$>5$ ans & 240 & 107 & 44,5 \\
Total & 480 & 212 & 44,1
\end{tabular}

\section{Tableau V}

Séroprévalence de l'infestation à Fasciola sp. chez les bovins N'Dama par troupeau villageois

\begin{tabular}{lccc} 
Région & Nb. troupeaux & Nb. positifs * & \% positifs \\
\hline BG & 11 & 7 & 63,6 \\
MG & 18 & 15 & 83,3 \\
HG & 25 & 23 & 92,0 \\
GF & 8 & 8 & 100 \\
Total & 62 & 53 & 85,4
\end{tabular}

* Un troupeau est considéré comme positif si au moins un animal du troupeau est positif

BG : Basse Guinée ; MG : Moyenne Guinée

HG : Haute Guinée ; GF : Guinée forestière

\section{DISCUSSION}

Les résultats sérologiques ont montré que la douve était endémique en Guinée. Presque un animal sur deux était ou avait été en contact avec le parasite : le test sérologique n'indiquait pas nécessairement une infestation active (4). La région Est était plus infestée que la région Ouest. Cette différence était significative et pouvait s'expliquer par la richesse de la zone en fleuves et en mares permanentes. Les fleuves Niger, Sankarani, Milo, Tinkisso et Diani traversent cette région. Au Bénin, la région le long du fleuve Niger est infestée de douves. Les bovins pâturent et s'abreuvent le long des fleuves et des mares pendant la saison sèche. Le gastéropode
Lymnaea natalensis, hôte intermédiaire de Fasciola gigantica, peut être observé le long des fleuves (11).

La majorité des troupeaux ont été positifs au test Elisa dans les différentes régions. Il est vraisemblable que les conditions nécessaires à l'évolution de la douve et de son hôte intermédiaire (Lymnaea natalensis) soient présentes dans les quatre régions (Basse Guinée, Moyenne Guinée, Haute Guinée et Guinée forestière).

Le sexe n'a pas eu d'effet sur l'infestation. Cette observation a été faite aussi au Nigeria (8).

Les animaux ont été infestés au cours de la première $(27,8$ p. 100) et de la deuxième année (52,3 p. 100). Le taux de séropositivité est resté plus ou moins stable (autour de 50 p. 100) pour les animaux de trois à cinq ans et plus. L'infestation des jeunes bovins pouvait être en relation avec la conduite du troupeau. En effet, ils pâturaient avec les adultes dès l'âge de deux à trois mois, pouvant ainsi être exposés à l'infestation de douves.

Il convient d'interpréter ces résultats sérologiques avec prudence car l'antigène utilisé pour le test était celui de $F$. hepatica. Néanmoins, l'immunodiagnostic de la fasciolose à $F$. hepatica peut mettre en évidence la fasciolose à $F$. gigantica à cause de la communauté antigénique entre ces deux espèces (7). Fasciola gigantica est l'agent causal de la fasciolose en Afrique de l'Ouest (11).

\section{CONCLUSION}

La fasciolose bovine existe en Guinée avec une forte infestation en Haute Guinée et Guinée forestière, formant la région Est de la Guinée. Cette parasitose ayant une importance sanitaire et économique, une enquête plus approfondie doit être conduite dans la région Est et, plus précisément, le long du fleuve Niger en Haute Guinée et du fleuve Diani en Guinée forestière, car ils sont les principaux fleuves de cette région. L'enquête sur ces sites permettra de déterminer l'ampleur épidémiologique de la maladie chez les bovins N'Dama vivant dans cette zone d'endémicité de la Guinée et aussi chez l'hôte intermédiaire de la fasciolose.

\section{Remerciements}

Nous remercions le comité de lecture et la rédaction de la Revue d'élevage et de médecine vétérinaire des pays tropicaux, ainsi que le Professeur G. Uilenberg, pour les remarques, suggestions et corrections qu'ils ont bien voulu apporter à cet article.

\section{BIBLIOGRAPHIE}

1. AWAD W.S., IBRAHIM A.K., SALIB F.A., 2009. Using indirect ELISA to assess different antigens for the serodiagnosis of Fasciola gigantica infection in cattle, sheep and donkeys. Res. vet. Sci., 86: 466-471.

2. BOUlARD C., BOUVRY M., ARGENTE G., 1985. Comparaison de la détection des foyers de fasciolose par test Elisa sur lactosérum et sur sérum, et par coproscopie. Ann. Rech. vét., 16 : 363-368.

3. DIAW O.T., SEYE M.M., SEYE M., SARR Y., VASSILLIADES G., 1994. L'immunodiagnostic de la fasciolose à Fasciola gigantica par la technique Elisa au Sénégal. Observations préliminaires chez deux agneaux. Revue Elev. Méd. vét. Pays trop., 47 : 291-294.

4. FAGBEMI B.O., ADERIBIGBE, GUOBADIA E.E., 1997. The use of monoclonal antibody for the immunodiagnosis of Fasciola gigantica infection in cattle. Vet. Parasitol., 68: 231-240.

5. FAGBEMI B.O., OBARISIAGBON I.O., 1990. Comparative evaluation of the enzyme-linked immunosorbent assay (ELISA) in the diagnosis of natural Fasciola gigantica infection in cattle. Vet. Quart., 12: 35-39. 
6. HANON J.B., DIAW O.T., SEYE M.M., JEAN G.J., CLAUDE S., 2009. Enquête de prévalence par immuno-diagnostic de la fasciolose des bovins (Fasciola gigantica) auprès des éleveurs peuls transhumants producteurs de lait dans la région du fleuve au Nord-Sénégal. Epidémiol. Santé Anim., 56 : 129-140.

7. MOLLOY J.B., ANDERSON G.R., FLETCHER T.I., LANDMANN J., KNIGHT B.C., 2005. Evaluation of a commercially available enzymelinked immunossorbent assay for detecting antibodies to Fasciola gigantica in cattle, sheep and buffaloes in Australia. Vet. Parasit., 130 207-212.

8. OGUNRINADE A.F., 1984. Bovine fascioliasis in Nigeria. VI. Parasitological characteristics of field infections. Revue Elev. Méd. vét Pays trop., 37: 299-303.
9. SHAHEEM H.I., KAMAL K.A., FARID Z., MANSOUR N., BOCTOR F.N., WOODY J., 1989. Dot-enzyme-linked immunosorbent assay (DotELISA) for the rapid diagnosis of human fascioliasis. J. Parasitol., 75: 549552 .

10. SYLLA A., 2010. Rapports annuels des saisies de foies dans les abattoirs de Guinée de 2005-2009. Conakry, Guinée, DNPR, 80 p.

11. YOUSSAO A.K.I., ASSOGBA M.N., 2002. Prévalence de la fasciolose bovine dans la vallée du fleuve Niger au Bénin. Revue Elev. Méd. vét. Pays trop., 55 : 105-108.

Accepté le 21.07.2011

\section{Summary}

Barry A.M., Keita S. Serological survey of bovine fasciolosis in Guinea

A serological survey on bovine fasciolosis was conducted from sera kept at the Veterinary Diagnosis Laboratory of the National Direction of Veterinary Services of Guinea. A total of 480 sera from N'Dama cattle were randomly selected and analyzed by the enzyme-linked immunosorbent assay (ELISA) technique. Results showed that $44.1 \%$ of sera presented antibodies to flukes with a rate of $35.5 \%$ in the Western region and $55.5 \%$ in the Eastern region of Guinea. Cattle of all ages were seropositive with rates ranging from 27.8 to $44.5 \%$.

Keywords: Cattle - Fasciola gigantica - Fasciolosis - ELISA Guinea.

\section{Resumen}

Barry A.M., Keita S. Encuesta serológica de la fasciolosis bovina en Guinea

Se realizó una encuesta serológica sobre la fasciolosis en Guinea, con sueros provenientes de la seroteca del laboratorio de diagnóstico veterinario de la Dirección nacional de los servicios veterinarios. Un total de 480 sueros provenientes de bovinos N'Dama fueron seleccionados al azar y analizados mediante la técnica de ensayo de inmunoabsorción ligado a enzimas (ELISA). Los resultados obtenidos pusieron en evidencia la presencia de anticuerpos de Fasciola en 44,1\% de los bovinos, con una tasa de 35,5\% en la región Oeste de Guinea y de $55,5 \%$ en la región Este. Los bovinos de todas las edades fueron seropositivos con tasas variables entre 27,8 y $44,5 \%$.

Palabras clave: Ganado bovino - Fasciola gigantica Fascíolosis - ELISA - Guinea. 